\title{
Cellular hypoxia promotes osteogenic differentiation of mesenchymal stem cells and bone defect healing via STAT3 signaling
}

\author{
Xin Yu ${ }^{1,2,3+}$, Qilong Wan ${ }^{2+}$, Xiaoling Ye ${ }^{1}$, Yuet Cheng ${ }^{1}$, Janak L. Pathak ${ }^{4^{*}}$ (D) and Zubing $\mathrm{Li}^{1,2^{*}}$
}

\footnotetext{
* Correspondence: janakpathak@ 163.com; lizubing@whu.edu.cn Xin Yu and Qilong Wan are share first authorship.

${ }^{4}$ Key Laboratory of Oral Medicine, Guangzhou Institute of Oral Disease, Affiliated Stomatology Hospital of Guangzhou Medical University, Guangzhou 510140, China ${ }^{1}$ The State Key Laboratory Breeding Base of Basic Science of Stomatology (Hubei-MOST) and Key Laboratory of Oral Biomedicine, Ministry of Education, School and Hospital of Stomatology, Wuhan University, 237 Luoyu Road, Wuhan 430079, China

Full list of author information is available at the end of the article
}

\begin{abstract}
Background: Hypoxia in the vicinity of bone defects triggers the osteogenic differentiation of precursor cells and promotes healing. The activation of STAT3 signaling in mesenchymal stem cells (MSCs) has similarly been reported to mediate bone regeneration. However, the interaction between hypoxia and STAT3 signaling in the osteogenic differentiation of precursor cells during bone defect healing is still unknown.

Methods: In this study, we assessed the impact of different durations of $\mathrm{CoCl}_{2}$-induced cellular hypoxia on the osteogenic differentiation of MSCs. Role of STAT3 signaling on hypoxia induced osteogenic differentiation was analyzed both in vitro and in vivo. The interaction between cellular hypoxia and STAT3 signaling in vivo was investigated in a mouse femoral bone defect model.

Results: The peak osteogenic differentiation and expression of vascular endothelial growth factor (VEGF) occurred after 3 days of hypoxia. Inhibiting STAT3 reversed this effect. Hypoxia enhanced the expression of hypoxia-inducible factor 1-alpha (HIF-1a) and STAT3 phosphorylation in MSCs. Histology and $\mu-C T$ results showed that $\mathrm{CoCl}_{2}$ treatment enhanced bone defect healing. Inhibiting STAT3 reduced this effect.

Immunohistochemistry results showed that $\mathrm{CoCl}_{2}$ treatment enhanced Hif- $1 \mathrm{a}, \mathrm{ALP}$ and pSTAT3 expression in cells present in the bone defect area and that inhibiting STAT3 reduced this effect.

Conclusions: The in vitro study revealed that the duration of hypoxia is crucial for osteogenic differentiation of precursor cells. The results from both the in vitro and in vivo studies show the role of STAT3 signaling in hypoxia-induced osteogenic differentiation of precursor cells and bone defect healing.
\end{abstract}

Keywords: Cellular hypoxia, Mesenchymal stem cells, STAT3-signaling, Osteogenic differentiation, Bone defect healing

\section{Introduction}

Bone defect healing is a complex process involving numerous cellular signaling pathways mediated by multiple factors, including hypoxia, inflammation and mechanical loading. Resolving the clinical issues of delayed bone defect healing and fracture nonunion requires a deeper understanding of these underlying cellular and molecular mechanisms.

(c) The Author(s). 2019 Open Access This article is distributed under the terms of the Creative Commons Attribution 4.0 International License (http://creativecommons.org/licenses/by/4.0/), which permits unrestricted use, distribution, and reproduction in any medium, provided you give appropriate credit to the original author(s) and the source, provide a link to the Creative Commons license, and indicate if changes were made. The Creative Commons Public Domain Dedication waiver (http://creativecommons.org/ publicdomain/zero/1.0/) applies to the data made available in this article, unless otherwise stated. 
In the early stage of bone defect healing, the hypoxia-inducible factor (HIF) regulatory pathway activates and further stimulates the expression of hypoxia response genes such as $\beta$-catenin and vascular endothelial growth factor (VEGF) [1-3]. Hypoxia in the vicinity of the bone defect triggers the osteogenic differentiation of precursor cells and promotes bone regeneration [2, 4-7]. Inducing hypoxia in precursor cells has been reported to enhance bone defect healing [3, 8-10]. Moreover, hypoxia promotes osteogenesis-angiogenesis coupling via VEGF signaling during bone defect healing [2, 11 , 12]. Recently, hypoxia-based bone tissue engineering approaches have been reported to facilitate bone defect healing $[12,13]$. However, the optimal duration of cellular hypoxia to achieve the maximum anabolic effect on osteogenic differentiation of MSCs is still unknown.

Signal transducer and activator of transcription 3 (STAT3) is a ubiquitously present transcription factor that mediates cell survival, proliferation and differentiation $[14,15]$. STAT3 signaling plays a vital role in bone homeostasis. Osteoblast- and osteoclastspecific knockout of STAT3 significantly reduces bone mineral density in mice [16, 17]. Cellular hypoxia upregulates STAT phosphorylation in MSCs [18]. Similarly, an increase in STAT3 phosphorylation has been reported during the osteogenic differentiation of human periosteal progenitors. Sun et al. recently reported on the beneficial role of STAT3 signaling in bone defect healing via suppression of regulatory $\mathrm{T}$ cell function [19]. Osteoblast- and osteocyte-specific inactivation of STAT3 also decreases mechanical load-driven bone formation [20]. Akermanite bioceramics, an osteoinductive bone graft, upregulates STAT3 signaling and promotes bone defect healing. Cellular hypoxia enhances the migration of MSCs via STAT3 signaling [18]. Moreover, the upregulation of JAK2, an upstream of STAT3 signaling, has been reported to induce osteogenic differentiation of progenitor cells and bone defect healing [21].

Multiple findings from the literature indicate a possible role of STAT3 signaling in hypoxia-induced bone defect healing. However, the interaction between hypoxia and STAT3 signaling during the osteogenic differentiation of precursor cells and bone defect healing still needs to be investigated.

In this study, we assessed the impact of different durations of cellular hypoxia on the osteogenic differentiation of mesenchymal stem cells (MSCs). Furthermore, we investigated the role of STAT3 signaling in hypoxia-mediated osteogenic differentiation of precursor cells and bone defect healing.

\section{Materials and methods}

Isolation and culture of mice MSCs

Six-week old C57BL/6 male mice were obtained from the Wuhan University Centre for Animal Experiments. The Medical Ethics Committee of the College and Hospital of Stomatology of Wuhan University approved all the animal experiments performed in this study. The mice MSCs were isolated and cultured as described previously [21, 22].

\section{Animal study}

All the animals in this study were treated according to internationally recognized guidelines on animal welfare. C57BL/6 mice $(n=75$, male, 8 weeks old with a weight between 20 and $25 \mathrm{~g}$ ) were used in this study. Femoral defects were created in 60 of the 
mice, while 15 mice were used as the blank control (i.e., no femoral injury). The 60 mice with femoral defects were randomly divided into 4 equal groups (control, $\mathrm{CoCl}_{2}$, $\mathrm{CoCl}_{2}+$ inhibitor, and inhibitor group) for the treatment protocol.

\section{Cell viability assay}

For cell viability analysis, MSCs $\left(3 \times 10^{3}\right.$ cells/well) were seeded in a 96-well plates (NEST Biotechnology). The full culture was for 7 days, with the cells being treated with $\mathrm{CoCl}_{2}\left(50 \mu \mathrm{M}\right.$, Sigma) for $1,3,5$ or 7 days. $\mathrm{CoCl}_{2}$ treatment in cell culture is an established method to create cellular hypoxia. CCK-8 reagent $(10 \mu \mathrm{l})$ was added to each well with incubation for $4 \mathrm{~h}$ at $37^{\circ} \mathrm{C}$. The media were transferred to a new 96-well plate, and the absorbance was measured using a Thermo Fisher Scientific Microplate Reader at $450 \mathrm{~nm}$.

HIF1- $\alpha$ is the main hypoxia-induced protein that gives further cellular signaling. $\mathrm{CoCl}_{2}$ treatment mimics hypoxia in cells by occupying the von Hippel-Lindau (VHL) protein-binding domain of HIF- $\alpha$, thus preventing its degradation [23]. In our previous study, we tested various concentrations of $\mathrm{CoCl}_{2}$ and found that $50 \mu \mathrm{M}$ robustly enhanced HIF- $1 \alpha$ protein expression in mouse MSCs [18]. Therefore, in this study, we choose $50 \mu \mathrm{M} \mathrm{CoCl}_{2}$ treatment in MSC culture to create a hypoxic environment.

\section{Gene expression analysis}

For quantitative real-time PCR assay, MSCs $\left(1 \times 10^{6}\right.$ cells/well) were seeded in 6-well plates and cultured in osteogenic medium consisting of $50 \mathrm{mg} / \mathrm{ml}$ ascorbic acid (Sigma), $10^{-8} \mathrm{~mol} / \mathrm{l}$ dexamethasone (Sigma) and $10 \mathrm{mM} \beta$-glycerophosphate (Sigma). The cells were treated with $50 \mu \mathrm{M} \mathrm{CoCl}_{2}$ for 1, 3, 5 and 7 days. The STAT3 inhibitor cryptotanshinone $(10 \mu \mathrm{M}$, Sigma) or DMSO was added to the cells in the appropriate groups for the duration of the culture. The dose of cryptotanshinone was chosen based on our previous research [18].

Tissue RNA was isolated from mice femurs with or without bone defects from each group ( $n=3$ per group) on day 7 . The samples from mice without bone defects were used as the blank control. The samples from mice that had bone defects but that did not receive $\mathrm{CoCl}_{2}$, or cryptotanshinone treatment were used as the negative control. All mice femurs were isolated and ground in the presence of liquid nitrogen. The tissue debris was soaked in guanidinium thiocyanate (GTC) buffer (Omega Bio-tek). Total RNA was extracted with EZNA Total RNA Kit (Omega). Total RNA $(1 \mu \mathrm{g})$ was reverse-transcribed to cDNA using the First Strand cDNA Synthesis Kit (Takara). Quantitative real-time PCR was performed using All-In-One qPCR Mix (GeneCopoeia) and a Bio-Rad CFX 96 Real-time PCR cylcer. Glyceraldehyde 3-phosphate dehydrogenase (GAPDH) was used as a housekeeping gene. All the primers used for quantitative real-time PCT are listed in Table 1.

\section{Western blot assay}

For western blot analysis, MSCs $\left(2 \times 10^{5}\right.$ cells/well) were seeded in 6-well plates and cultured in osteogenic media. Cells were treated with $\mathrm{CoCl}_{2}(50 \mu \mathrm{M})$ for 1, 3, 5 and 7 days. Cryptotanshinone $(10 \mu \mathrm{M})$ or DMSO was added to the cells in the appropriate groups for the duration of the culture. Cell lysates were extracted on day $7[18,22]$. 
Table 1 Primer sequences

\begin{tabular}{|c|c|c|c|}
\hline Gene & Acc. No & Primer sequence $\left(5^{\prime} \rightarrow 3^{\prime}\right)$ & Size $(b p)$ \\
\hline \multirow[t]{2}{*}{ Hif-1a } & NM_176958 & F: GTCCCAGCTACGAAGTTACAGC & 136 \\
\hline & & R: CAGTGCAGGATACACAAGGTTT & \\
\hline \multirow[t]{2}{*}{ Alp } & NM_001081082 & F: GGCCATCTAGGACCGGAGA & 79 \\
\hline & & R: TGTCCACGTTGTATGTCTTGG & \\
\hline \multirow[t]{2}{*}{ Opn } & NM_001204203 & F: ATCTCACCATTCGGATGAGTCT & 79 \\
\hline & & R: TGTAGGGACGATTGGAGTGAAA & \\
\hline \multirow[t]{2}{*}{ Runx2 } & NM_001146038 & F: GACTGTGGTTACCGTCATGGC & 84 \\
\hline & & R: ACTTGGTIITCATAACAGCGGA & \\
\hline \multirow[t]{2}{*}{ Collar } & NM_007742 & F: GCTCCTCTTAGGGGCCACT & 91 \\
\hline & & R: ATTGGGGACCCTTAGGCCAT & \\
\hline \multirow[t]{2}{*}{ Osx } & NM_146065 & F: ATCATTGCAGATCAAACGCCT & 135 \\
\hline & & R: AGCACCCTIITTCTCATCGTC & \\
\hline \multirow[t]{2}{*}{ Ocn } & NM_031368 & F: CACTCCTCGCCCTATTGGC & 112 \\
\hline & & R: CCCTCCTGCTTGGACACAAAG' & \\
\hline \multirow[t]{2}{*}{ Opn } & NM_026493 & F: GAAGAGCAAAAAGCGAAACTGG & 153 \\
\hline & & R: TTGGCTGCTTGGTGGAATGT & \\
\hline \multirow[t]{2}{*}{ Vegf } & NM_005429 & F: GAGGAGCAGTTACGGTCTGTG & 96 \\
\hline & & R: TCCTTTCCTTAGCTGACACTTGT & \\
\hline \multirow[t]{2}{*}{ Gapdh } & NM_008085 & F: AATGGATTTGGACGCATTGGT & 213 \\
\hline & & R: TTTGCACTGGTACGTGTTGAT & \\
\hline
\end{tabular}

Total protein was estimated using the BCA Protein Assay (Thermo Scientific). Total protein $(20 \mu \mathrm{g})$ was separated by $10 \%$ SDS-PAGE (Biotech) and transferred to polyvinylidene difluoride membrane (Roche). The membranes were blocked with 5\% skimmed milk (Biosharp) absorbed in 10\% tris-buffered saline with 0.1\% tween 20 (TBST; Gibco) at room temperature for $1 \mathrm{~h}$. Then, the membranes were incubated on a shaker for $8 \mathrm{~h}$ at $4{ }^{\circ} \mathrm{C}$ with one of the primary antibodies: anti-HIF-1 $\alpha$ (Santa), anti-ALP (Abcam), anti-Osx (Abcam), anti-Runx2 (Abcam), anti-Col1 $\alpha 1$ (Santa) and anti-GAPDH (Protech). The membranes were then incubated with secondary antibody (Abbkine) and absorbed in TBST for $1 \mathrm{~h}$ at room temperature. Blots were visualized, and the relative density of each blot was determined using Image J software 1.49 (NID).

\section{ALP staining and ALP activity}

For ALP staining and activity, MSCs $\left(1 \times 10^{5}\right.$ cells/well $)$ were seeded in 24-well plates and cultured in osteogenic medium. Cells were treated with $\mathrm{CoCl}_{2}(50 \mu \mathrm{M})$ for $1,3,5$ and 7 days. Cryptotanshinone $(10 \mu \mathrm{M})$ or DMSO was added to the cells in the appropriate groups for the duration of the culture. The ALP staining and activity measurements were conducted on day $7[18,22]$.

\section{Alizarin red assay}

To analyze the mineralized matrix, MSCs $\left(1 \times 10^{5}\right.$ cells/well $)$ were seeded in 24-well plates and cultured in osteogenic medium. Cells were treated with $\mathrm{CoCl}_{2}(50 \mu \mathrm{M})$ for 1 , 3, 5 and 7 days. Cryptotanshinone $(10 \mu \mathrm{M})$ or DMSO was added to the cells in the 
appropriate groups for the duration of the culture. All the cultures were continued till day 14 in presence of osteogenic medium. The matrix-mineralized nodules were stained with alizarin red on day 14. Cultures were washed 3 times with PBS, fixed with 95\% ethanol for $15 \mathrm{~min}$, and washed 3 times with distilled water. The cells were stained with $0.1 \%$ alizarin red S (Sigma) dissolved in $0.1 \mathrm{M}$ Tris- $\mathrm{HCl}$ buffer for $30 \mathrm{~min}$. A light microscope was used to visualize the alizarin red-stained mineralized matrix. Quantification involved dissolving the mineralized matrix with $1 \%$ cetylpyridinium chloride and measuring the absorbance of the dissolved matrix at $562 \mathrm{~nm}$ using a microplate reader.

\section{In vivo bone defect healing}

The femoral defects were prepared based on findings from our previous research [24]. The anesthetic agent was a mixture of $1.5 \mathrm{mg} / \mathrm{ml}$ xylazine and $10 \mathrm{mg} / \mathrm{ml}$ ketamine. It was intraperitoneally injected at a dose of $0.1 \mathrm{ml} / 10 \mathrm{~g}$ body weight. A longitudinal lateral thigh incision was made to expose the femur. A perforated defect was drilled in the middle of the femur using a $0.8 \mathrm{~mm}$ straight shank twist drill (GB/T6135.2, Shanghai Tool Works) at $3000 \mathrm{rpm}$ attached to a grinder set (P-500-6A, Slite). Then a 1.4-mm straight shank twist drill (Shanghai Tool Works) was used to extend the defect. To avoid thermal injury, saline irrigation was used. The surgical area was flushed with saline solution to remove the bone chips. The incision was closed in layers with sterile silk suture. For postoperative analgesia, $50 \mu \mathrm{l}$ buprenorphine hydrochloride at a concentration of $0.04 \mathrm{mg} / \mathrm{ml}$ was injected every $12 \mathrm{~h}$ subcutaneously for 2 days.

Mice in the blank control group received no treatment. The treatment consisted of daily intraperitoneal injection with: $\mathrm{CoCl}_{2}\left(13.5 \mathrm{mg} / \mathrm{kg}\right.$ body weight) for the $\mathrm{CoCl}_{2}$ and $\mathrm{CoCl}_{2}+$ inhibitor groups; cryptotanshinone $\left(5 \mathrm{mg} / \mathrm{kg}\right.$ body weight) for the $\mathrm{CoCl}_{2}+$ inhibitor and inhibitor groups; or 0.1\% DMSO absorbed in PBS (control group).

In each group, 5 mice were randomly assigned to each time point (1, 3 or 5 weeks post operation). For RNA isolation and qPCR analysis, samples from 15 mice (3 mice/ group, total 5 groups) were used as described in the section on gene expression analysis. Mice were killed 1, 3 or 5 weeks after surgery. Femora were fully dissected and fixed in $4 \%$ PFA for $48 \mathrm{~h}$.

\section{Micro-CT analysis}

The femora were fixed in $4 \%$ paraformaldehyde and scanned using a $\mu$-CT 50 imaging system (Scanco Medical). The tube was set at $70 \mathrm{kV}, 85 \mathrm{~mA}$ with $20 \mu \mathrm{M}$ resolution. Newly formed bone volume and trabecular parameters were quantified in a $2-\mathrm{mm}^{3}$ cube that completely contained the bone defect area.

\section{Histology and immunohistochemistry study}

Fixed bone tissues ( $n=5$ /group) were embedded in paraffin and sliced into $5 \mu \mathrm{M}$ thick tissue sections using a microtome (Thermo Fisher Scientific). The tissue sections were deparaffinized and stained with hematoxylin and eosin (H\&E). Newly formed bone and osteoids were visualized under a light microscope. For immunohistochemistry, tissue sections were stained according to the standard protocol [22]. The primary antibodies were anti-HIF-1 $\alpha$ (CST, 1:400 dilution), anti-pSTAT3 (CST, 1:200 dilution), anti-ALP 
(Abcam, 1:400 dilution). The universal immunoperoxidase (HRP) ABC kit (ZSGB bio) was used to visualize the secondary antibody.

\section{Statistical analysis}

All the in vitro and in vivo experiments were performed 5 times $(n=5)$. Data analysis was performed using a one-way analysis of variance (ANOVA) followed by Bonferroni's multiple comparison test using GraphPad Prism 7.0 software. In all cases, the selected significance level was $p<0.05$.

\section{Results}

Cellular hypoxia suppressed MSC proliferation but enhanced osteogenic differentiation

The hypoxia simulated by $\mathrm{CoCl}_{2}$ treatment significantly suppressed MSC proliferation. One day of hypoxia (i.e., 1 day of $\mathrm{CoCl}_{2}$ treatment followed by 6 days of incubation without $\mathrm{CoCl}_{2}$ ) did not show an impact on MSC proliferation after 1, 3 or 5 days of culture, but 1.34-fold inhibition was observed on day 7 (Fig. 1a). Interestingly, hypoxia for 3, 5 and 7 days showed similar inhibitory effect on cell proliferation after 5 and 7 days of culture (Fig. 1a).

Osteogenic gene expression, ALP staining and ALP activity in MSCs were analyzed on day 7. Hypoxia for 3 days respectively upregulated Col1 1 1, Runx2, Alp, Osx, Opn, Ocn and Vegf gene expression by 3.12-, 3.35-, 4.12-, 14.29-, 8.35-, 12.1- and 2.61-fold compared to the control group (Fig. 1b-j). Hypoxia for 5 days enhanced only Ocn and Vegf gene expression (respectively by 9.07 - and 1.75- fold compared to the control group). Hypoxia for 1 day enhanced Vegf gene expression by 2.05 -fold compared to the control group, but did not affect the expressions of other osteogenic markers (Fig. 1h). Interestingly, continuous simulation of hypoxia for 7 days did not affect all the expressions of all the osteogenic markers tested (Fig. 1b-j).

Hypoxia for 3 days yielded the strongest ALP and alizarin red staining (Fig. 2a and c). Similarly, hypoxia for 3 days enhanced ALP activity by 2.92- fold compared to the

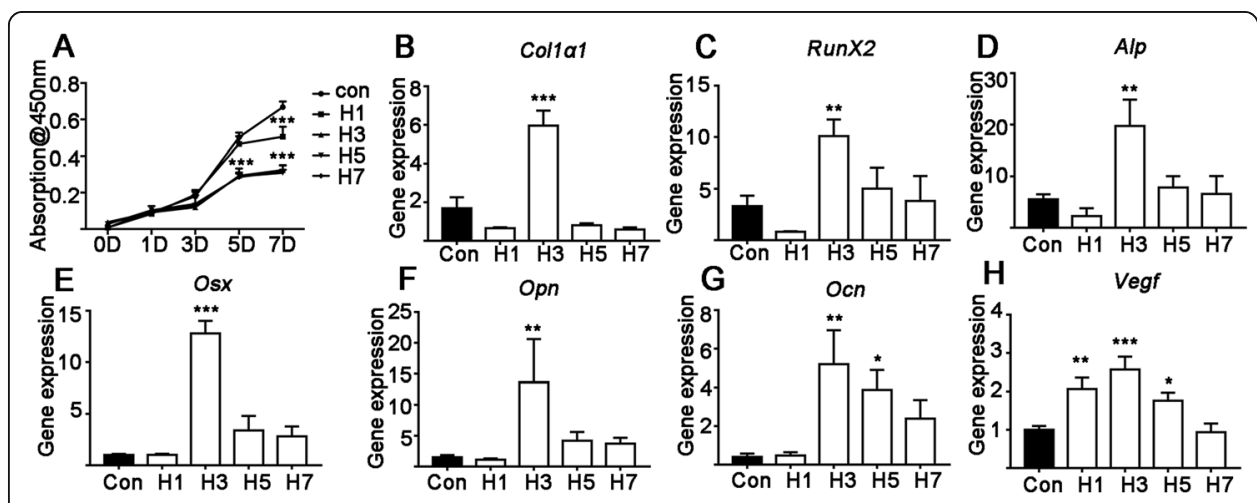

Fig. 1 The effect of continuous hypoxia for 1, 3, 5 and 7 days on MSC proliferation and gene expression of osteogenic markers. a MSC proliferation on days 1, 3, 5 and 7 of culture including 1, 3, 5 or 7 days of hypoxia. b-h Osteogenic and Vegf gene expression on day 7. Data are the means \pm SD from 5 independent experiments, $n=5$. Significant effect of the treatment: ${ }^{*} p<0.05,{ }^{* *} p<0.01$ and ${ }^{* * *} p<0.001 . \mathrm{H} 1, \mathrm{H} 3, \mathrm{H} 5$ and H7 represent hypoxia for 1, 3, 5 and 7, respectively, whule 0D, 1D, 3D, 5D and 7D represent 0, 1, 3, 5 or 7 total days of culture, respectively. Col1a1: collagen I alpha1, Runx2: Runt-related transcription factor 2, Alp: alkaline phosphatase, Osx: osterix, Ocn: osteocalcin and Vegf: vascular endothelial growth factor 


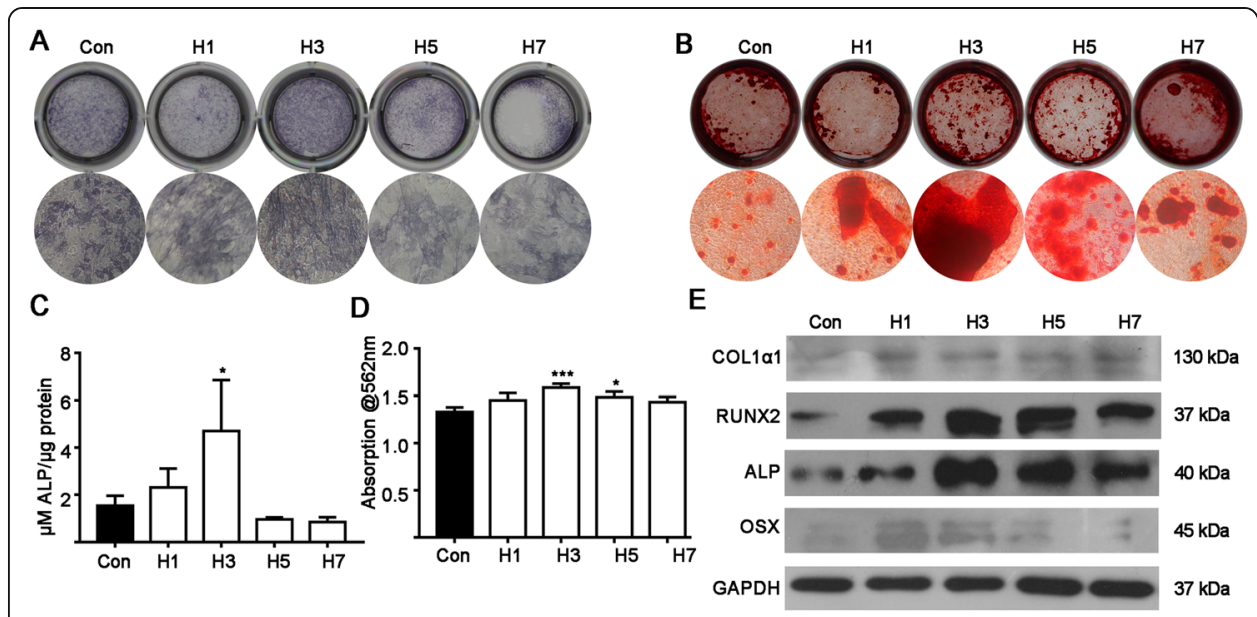

Fig. 2 The effect of continuous hypoxia for 1, 3, 5 and 7 days on ALP activity and matrix mineralization. a ALP staining on day 7. b Matrix mineralization (alizarin red staining) on day 14. c ALP activity on day 7. d Quantitative analysis of alizarin red staining. e Osteogenic differentiation marker (protein) expression. Data from quantitative analysis are the means \pm SD from 5 independent experiments, $n=5$. Significant effect of the treatment: ${ }^{*} p<0.05,{ }^{* *} p<0.01$ and ${ }^{* * *} p<0.001$

control group (Fig. 2c). Quantification of the mineralized matrix showed that hypoxia for 3 and 5 days respectively promoted matrix mineralization by 1.18-, and 1.09-fold compared to the control group (Fig. 2d).

COL1 $\alpha 1$, OSX, RUNX2, and ALP protein expressions were analyzed using western blot assay on day 7 . Hypoxia for $1,3,5$ and 7 days respectively enhanced COL $1 \alpha 1$ protein expression by 1.50-, 1.55-, 1.41- and 1.47- fold (Fig. 2e and Additional file 1: Figure S1A). Hypoxia for 1, 3, 5 and 7 days respectively enhanced RUNX2 protein by 2.67-, 4.05-, 2.97and 2.29- fold (Fig. 2e and Additional file 1: Figure S1A). Hypoxia for 1, 3, 5 and 7 days respectively enhanced ALP protein expression by 1.81-, 4.97-, 2.68- and 1.91- fold (Fig. 2e and Additional file 1: Figure S1A). Similarly, hypoxia for 1, 3, 5 and 7 days respectively enhanced OSX protein expression by 1.84-, 2.71-, 2.25- and 2.08- fold (Fig. 2e and Additional file 1: Figure S1A). Hypoxia for 3 days showed the highest effect on osteogenic marker protein expression, which concurs with the results for mRNA expression.

\section{STAT3 inhibitor reversed hypoxia-induced STAT3 phosphorylation and osteogenic differentiation}

Cellular hypoxia enhanced HIF- $1 \alpha$ expression by 1.81 -fold. The STAT3 inhibitor did not alter hypoxia-induced HIF-1 $\alpha$ expression (Fig. 3a and Additional file 1: Figure S2A). Hypoxia did not affect the total STAT3 expression (Fig. 3a). Hypoxia robustly enhanced (5.46-fold) STAT3 phosphorylation and the STAT3 inhibitor reduced this effect by 3.10-fold (Fig. 3a and Additional file 1: Figure S2B).

Since hypoxia for 3 days showed the highest effect on the osteogenic differentiation of MSCs, we choose this culture condition to analyze the effect of STAT3 inhibitor on hypoxia-induced osteogenic differentiation. STAT3 inhibitor respectively suppressed hypoxia-induced Col1 1 1, Runx2, Alp, Osx, Opn, Ocn and Vegf gene expression by 6.13-, 4.87-, 5.67-, 6.56-, 4.31-, 5.41- and 2.63-fold (Fig. 3b-h). STAT3 inhibitor alone did not affect the expression of osteogenic genes compared to the control group (Fig. 3b-h). 


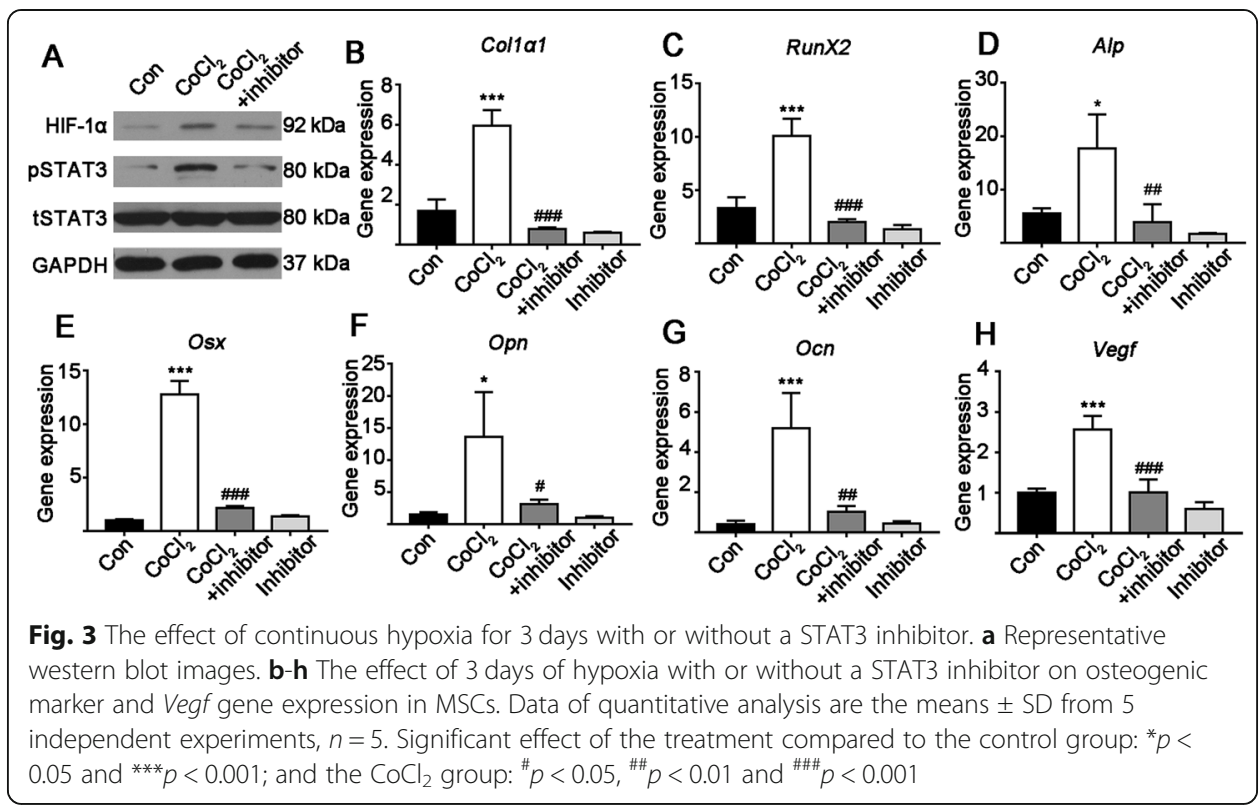

STAT3 inhibitor reduced hypoxia-induced ALP protein expression and ALP activity (5.38-fold; Fig. 4a and c). STAT3 inhibitor strongly reduced (2.37-fold) hypoxiainduced matrix mineralization (Fig. $4 \mathrm{~b}$ and d). Similarly, STAT3 inhibitor reduced matrix mineralization by 2.08 - and 4.51 -fold respectively compared to the results for the $\mathrm{CoCl}_{2}+$ inhibitor and control groups.

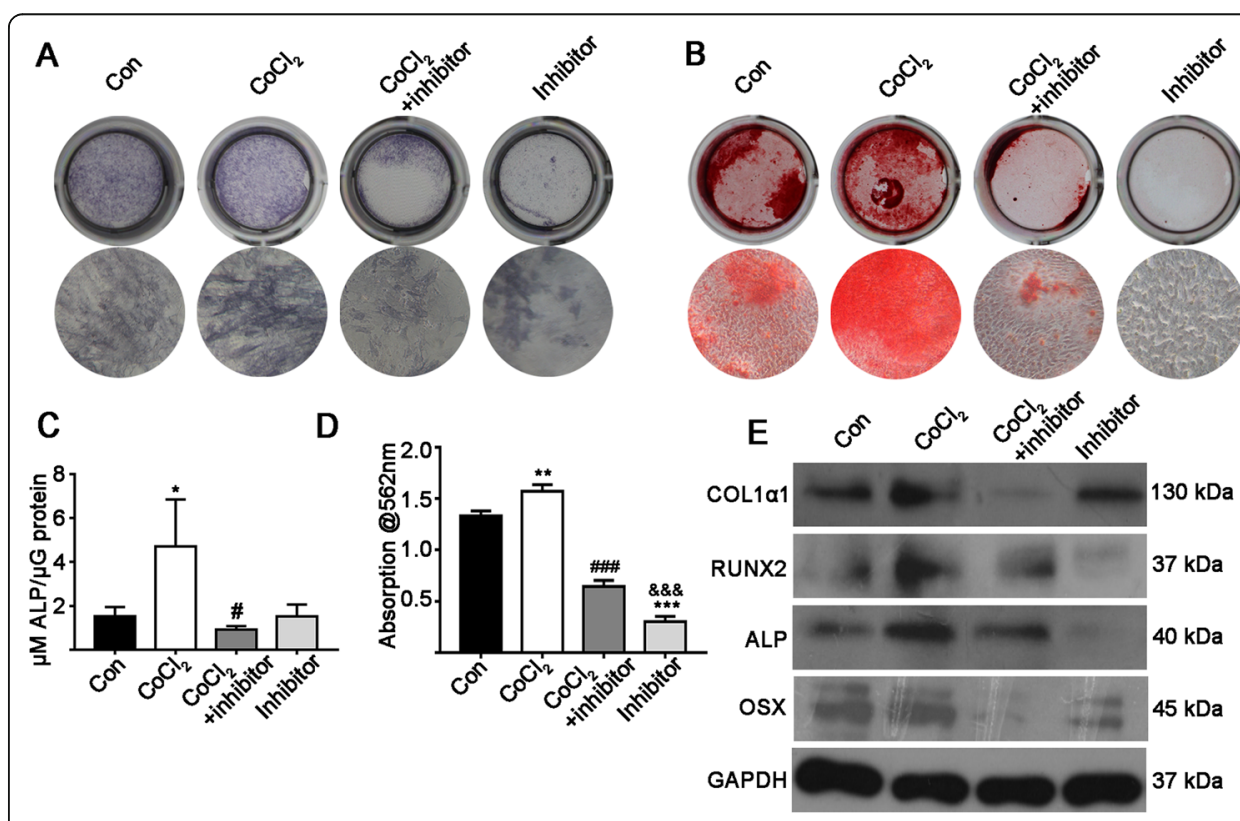

Fig. 4 The effect of continuous hypoxia for 3 days on days 1, 3, 5 and 7 of culture with or without a STAT3 inhibitor. a ALP staining on day 7. b Matrix mineralization (alizarin red staining) on day 14. c ALP activity on day 7. d Quantitative analysis of alizarin red staining. e Osteogenic differentiation marker (protein) expression. Data of quantitative analysis are the means \pm SD from 5 independent experiments, $n=5$. Significant effect of the treatment compared to the control group: ${ }^{*} p<0.05,{ }^{* *} p<0.01$ and ${ }^{* * *} p<0.001$; the $\mathrm{CoCl}_{2}$ group: ${ }^{\#} p<0.01,{ }^{\# \# \#} p<0.001$; and the $\mathrm{CoCl}_{2}+$ inhibitor group: ${ }^{\& \&} \&<0.001$. Inhibitor: STAT3 inhibitor 
Western blot data analysis showed that STAT3 inhibitor respectively reduced hypoxia-induced COL1 $\alpha 1$, RUNX2, ALP and OSX protein expressions by 4.56-, 1.67-, 1.34- and 1.78-fold (Fig. 3e and Additional file 1: Figure S3A-D). STAT3 inhibitor reduced OSX protein expression by 1.89 -fold compared to the control group (Additional file 1: Figure S3D). However, STAT3 inhibitor did not affect the expressions of the other osteogenic proteins tested compared to the control group. RUNX2, ALP and OSX protein expression in STAT3 inhibitor group were respectively suppressed by 3.23-, 3.02- and 2.35-fold compared to the hypoxia + STAT3 inhibitor group (Additional file 1: Figure S3B-D).

\section{Hypoxia enhanced bone regeneration and STAT3 inhibitor impaired this effect}

Histological images showed more newly formed bone in the bone defect area at week 3 in the $\mathrm{CoCl}_{2}$ group compared to the images for the control, hypoxia + STAT3 inhibitor, and STAT3 inhibitor groups (Fig. 5a). Similarly, the bone defect area was filled with newly formed bone in the $\mathrm{CoCl}_{2}$ group at week 5 . However, a clear bone defect gap was observed in the control, hypoxia + STAT3 inhibitor, and STAT3 inhibitor groups (Fig. 5a).

Hypoxia upregulated Hif-1a and Alp mRNA expression in bone defect femora and STAT3 inhibition reversed this effect

To investigate the possible interaction between hypoxia and STAT3 signaling during osteogenesis and bone defect healing, we analyzed Hif- $1 \alpha$ and Alp mRNA expression in

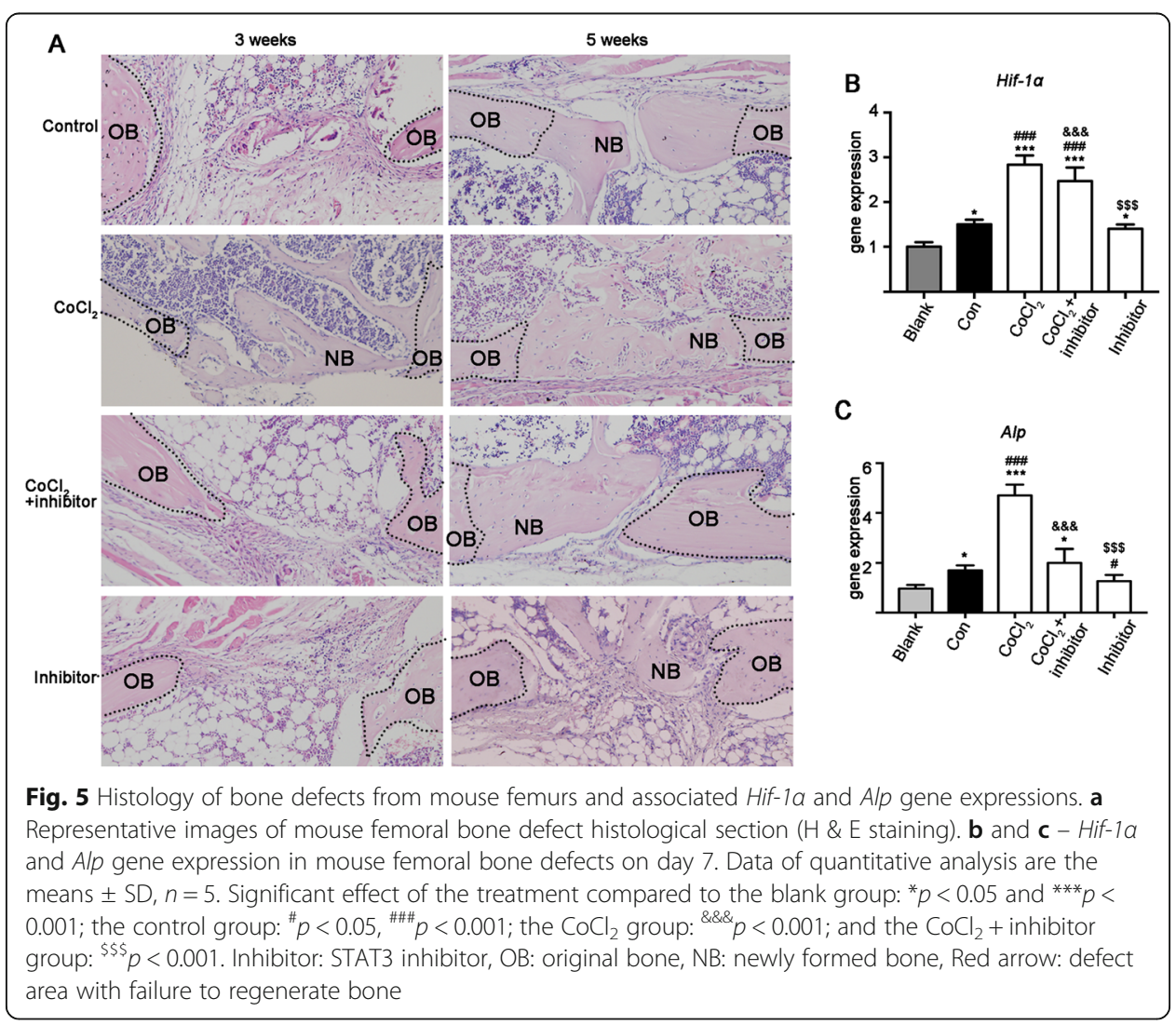


mice femoral bone defects treated with $\mathrm{CoCl}_{2}$ and/or STAT3 inhibitor. Hif-1 $\alpha$ and Alp mRNA expression were upregulated in the femurs of all the bone defect groups compared to the results for the blank control group (Fig. $5 \mathrm{~b}$ and c). $\mathrm{CoCl}_{2}$-induced hypoxia further upregulated Hif- $1 \alpha$ and Alp expression by 1.81 - and 2.77-fold, respectively (Fig. 5b and c). STAT3 inhibitor reduced hypoxia-induced Hif- $1 \alpha$ and Alp expression by 1.15 - and 2.30-fold, respectively (Fig. $5 \mathrm{~b}$ and c). The STAT3 inhibitor did not affect Hif- $1 \alpha$ expression but suppressed the Alp expression by 1.31-fold compared to the control group (Fig. 5c).

\section{$\mathrm{CoCl}_{2}$-simulated hypoxia promoted bone defect healing and STAT3 inhibitor reversed} this effect

$\mu$-CT and X-ray images showed that $\mathrm{CoCl}_{2}$ promoted femoral bone defect healing at week 3 and 5 compared to the control group (Fig. 6a and Additional file 1: Figure S4). Interestingly, the STAT3 inhibitor reversed hypoxia-induced bone defect healing at

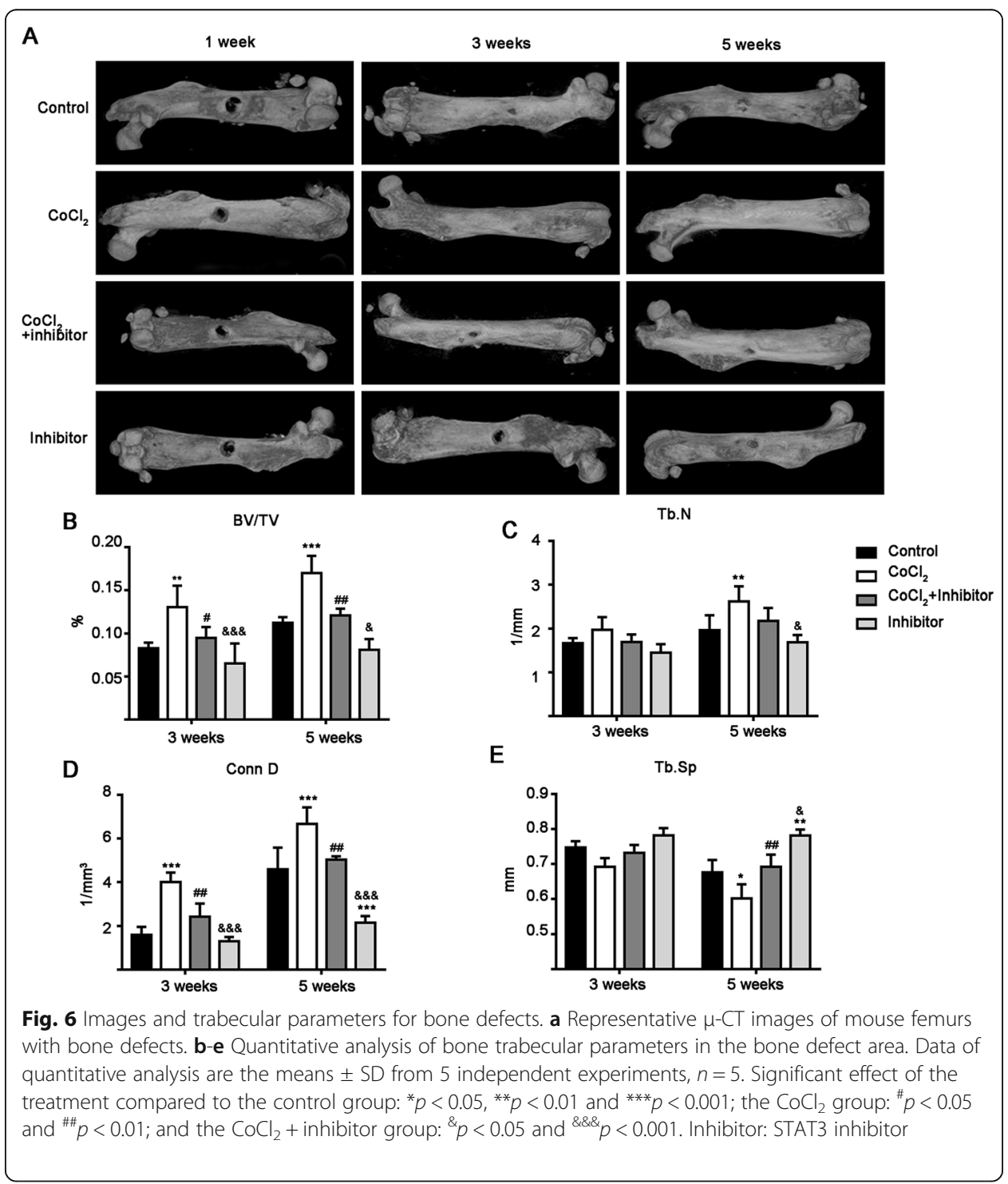


week 3 and 5 (Fig. 6a and Additional file 1: Figure S4). Moreover, STAT3 inhibitor reduced bone defect healing compared to the control, $\mathrm{CoCl}_{2}$ and $\mathrm{CoCl}_{2}+$ STAT3 inhibitor groups (Fig. 6a and Additional file 1: Figure S4).

Similar effects of $\mathrm{CoCl}_{2}$ and STAT3 inhibitor were shown by the newly formed bone trabecular parameters at week 3 and week 5 (Fig. $6 \mathrm{~b}-\mathrm{e}$ ). $\mathrm{CoCl}_{2}$ treatment enhanced BV/TV and Conn D levels by 1.51- and 2.44-fold, respectively, at week 3 compared to the control. STAT3 inhibitor reduced the $\mathrm{CoCl}_{2}$-induced impact on BV/TV and Conn D levels by 1.37- and 1.64-fold, respectively (Fig. $6 \mathrm{~b}$ and d). Similarly, STAT3 inhibitor reduced BV/TV and Conn D levels by 1.28- and 1.27-fold, respectively, compared to the control group at week 3 (Fig. 6a and d). $\mathrm{CoCl}_{2}$ treatment enhanced BV/TV, Tb. N and Conn D levels by 1.49-, 1.45- and 1.46-fold, respectively, at week 5 compared to the control group (Fig. $6 \mathrm{~b}-\mathrm{e}$ ). STAT3 inhibitor reduced the $\mathrm{CoCl}_{2}$-induced impact on BV/TV and Conn D levels by 1.38- and 1.31- fold, respectively, at week 5 (Fig. 6b-e). Moreover, STAT3 inhibitor reduced Conn D levels by 2.08-fold compared to the control (Fig. 6b-e), and reduced BV/TV, Tb. N, and Conn D levels by 1.49-, 1.25- and 2.27-fold, respectively, compared to those for the $\mathrm{CoCl}_{2}+$ inhibitor group at week 5 (Fig. 6b-e). Hypoxia suppressed Tb. Sp levels by 1.38 -fold at week 5 compared to the control group (Fig. 6e). STAT3 inhibitor reversed hypoxia-mediated suppression at week 5 (Fig. 6e). Moreover, the STAT3 inhibitor group enhanced Tb. Sp levels by 1.36and 1.30 -fold at week 5 compared to the control and $\mathrm{CoCl}_{2}+$ inhibitor groups, respectively (Fig. 6e).

Hypoxia upregulated HIF-1a, PSTAT3 and ALP protein expression in the bone defect area and STAT3 inhibitor reversed this effect

Immunohistochemistry images showed stronger immunostaining (brown and light brown color) of HIF-1 $\alpha$, p-STAT3 and ALP at week 3 compared to the staining at week 5 in all the groups tested (Fig. $7 \mathrm{a}-\mathrm{C}$ ). The $\mathrm{CoCl}_{2}$-treated group showed stronger immunostaining of HIF-1 $\alpha$, p-STAT3 and ALP compared to all other groups at week 3 and 5 .

Quantitative analysis of the immunohistochemistry of tissue section showed 3.32and 1.74-fold higher expression of HIF- $1 \alpha$ in the hypoxic group compared to the control group at week 3 and 5, respectively (Fig. 7a and d). STAT3 inhibitor did not affect $\mathrm{CoCl}_{2}$-induced HIF- $1 \alpha$ expression at week 3 and 5 . The $\mathrm{CoCl}_{2}$ group showed 1.87- and 2.85 -fold higher expression of pSTAT3 compared to the control group at week 3 and 5, respectively (Fig. 7a and d). STAT3 inhibitor reversed the hypoxia-induced pSTAT3 upregulation at week 3 and 5. Similarly, the $\mathrm{CoCl}_{2}$-group showed 2.02- and 1.97-fold higher expression of ALP compared to the control group at week 3 and 5, respectively (Fig. 7a and d). STAT3 inhibitor reduced the hypoxia-induced ALP expression by 1.73and 1.70 -fold at week 3 and 5 , respectively.

\section{Discussion}

The crosstalk (interaction) between cellular hypoxia and STAT3 signaling during bone defect healing has not been reported on yet. In this study, we found that short-term (3day) cellular hypoxia enhanced the osteogenic differentiation of MSCs and bone defect healing, and that inhibition of STAT3 signaling reversed this effect. Moreover, cellular 


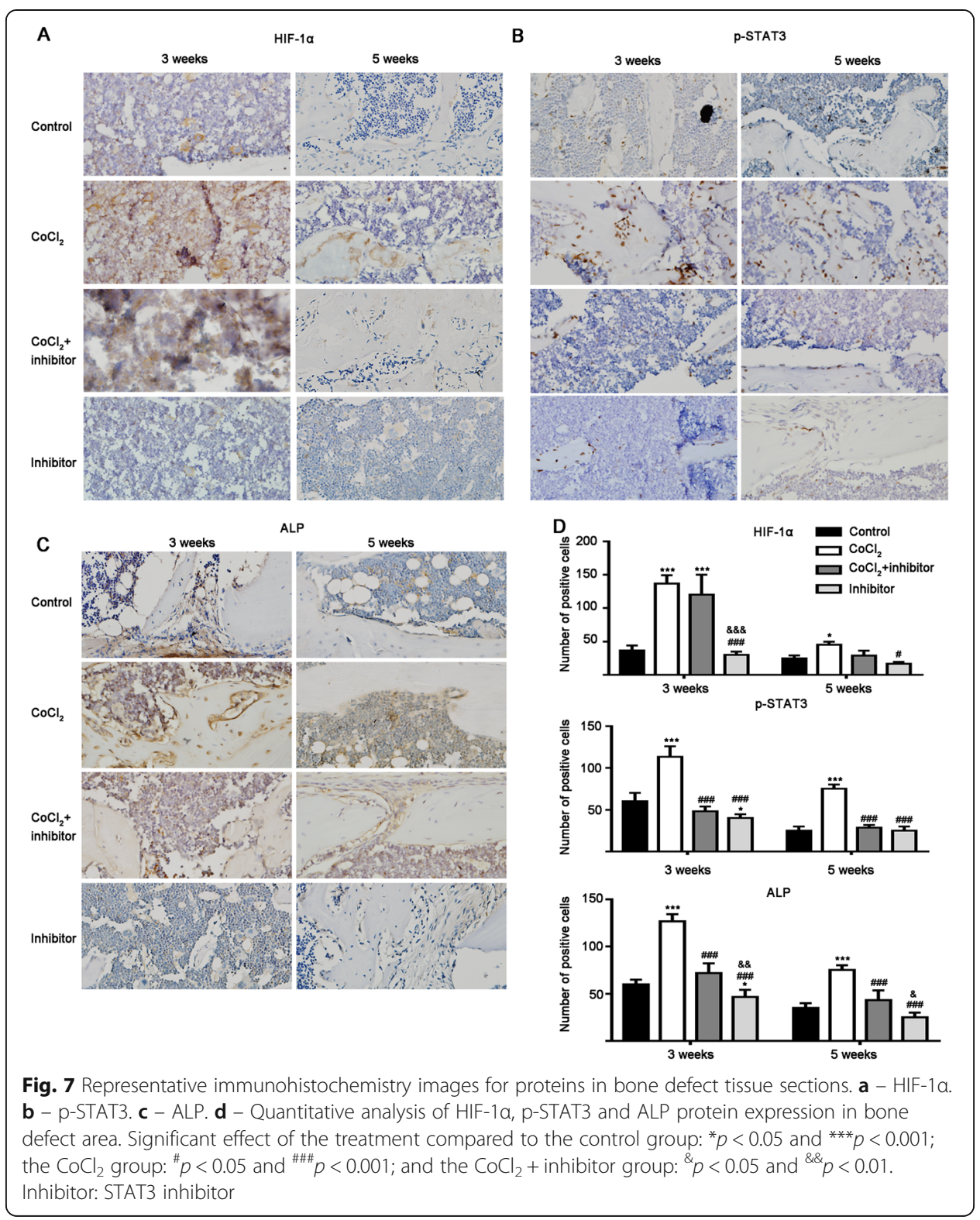

hypoxia upregulated Vegf, HIF-1 $\alpha$ and pSTAT3 expression during in vitro osteogenic differentiation and bone defect healing. The STAT3 inhibitor neutralized this effect. These findings suggest that the interaction between hypoxia and STAT3 signaling is significant in bone defect healing.

We analyzed the effects of the different durations of hypoxia on MSC proliferation during a 7-day culture. Hypoxia for 3, 5 and 7 days resulted in a similar level of inhibition on MSCs proliferation on day 7. The effects of different duration of hypoxia on osteogenic marker expression was also evaluated on day 7. Three days of hypoxia robustly enhanced the expressions of most osteogenic markers at the mRNA and protein levels on day 7, while 1, 5 and 7 days of hypoxia did not have this effect (Fig. 1). This result was further confirmed by the highest ALP activity (on day 7) and matrix mineralization (on day 14) being found for MSCs exposed to hypoxic conditions for 3 days (Fig. 2). 
It is well known that hypoxia in early-stage bone defect healing triggers the healing process. However, it has also been reported that continuous hypoxia inhibits osteogenic differentiation of precursor cells $[25,26]$ via activation of Notch1 signaling [26] and inhibition of Runx2 [27]. Osathanon et al. had reported that continuous treatment with $\mathrm{CoCl}_{2}(50 \mu \mathrm{M})$ for 7 days does not affect OCN gene expression, ALP activity or matrix mineralization in human periodontal ligament stem cell culture [25]. Xu et al. reported the inhibitory effect of continuous hypoxia for 7 days or more on the osteogenic differentiation of MSCs [26]. Moreover, Genetos et al. reported that $48 \mathrm{~h}$ hypoxia activates Wnt signaling and suppresses sclerostin expression in osteoblasts [28]. Activated Wnt signaling induces osteogenesis and high sclerostin expression inhibits it.

During the early stage of bone defect healing, hypoxia enhances the migration of osteogenic and angiogenic precursor cells as well as osteogenesis and angiogenesis. Newly formed vessels around the bone defect eliminate the hypoxic condition and the healing process continues. During embryo development, hypoxia is essential for vascularization of the placenta and embryo. Similarly, endochondral ossification during fetal bone development requires a hypoxic environment. Hypoxia-mediated endochondral ossification also plays a role in large size bone defect healing [29]. Tissue engineering techniques utilizing hypoxia are being developed to repair large bone and cartilage defects. Hypoxic conditions (3\% oxygen) in bioreactors enhance chondrogenesis and cartilage matrix component formation [30]. Moreover, intermittent hypoxia has been reported to promote hippocampal neurogenesis and provide antidepressant-like effects in adult rats [31].

The bone defect healing process also indicates the importance of the period of hypoxia. In this study, continuous hypoxia inhibited most osteogenic differentiation markers, including Runx2. Our results showed that hypoxia for too short (1 day) or too long ( 5 and 7 days) a period failed but for 3 days robustly enhanced the osteogenic differentiation of precursor cells. This suggests hypoxia has a crucial optimal duration that induces bone regeneration in the healing process.

The activation of STAT3 signaling has been reported to enhance the osteogenic differentiation of precursor cells and bone defect healing [19, 32]. Gao et al. had reported that hypoxia enhances STAT3 signaling in synovial fibroblasts [33]. However, a few studies have investigated the interaction between hypoxia and STAT3 signaling during the osteogenic differentiation of precursor cells. In this study, $\mathrm{CoCl}_{2}$ treatment enhanced HIF- $1 \alpha$ protein expression in MSCs (Fig. 3a), which indicates that the $\mathrm{CoCl}_{2}$ treatment in MSC culture could induce cellular hypoxia. Since cellular hypoxia enhanced STAT3 phosphorylation and a STAT3 inhibitor reduced this effect (Fig. 3a), we further investigated the effect of the STAT3 inhibitor on hypoxia-induced osteogenic differentiation and bone defect healing. Interestingly, inhibition of STAT3 dramatically reversed the stimulatory effect of hypoxia on osteogenic differentiation of MSCs (Figs. 3 and 4). These findings indicate that hypoxia-mediated activation of STAT3 promotes osteogenic differentiation of MSCs. This is the first study to report on STAT3mediated osteogenic differentiation of MSCs and matrix mineralization under hypoxic conditions.

Osteogenesis-angiogenesis coupling plays a vital role in bone regeneration during bone defect healing [11, 34]. VEGF is a known pro-angiogenic as well as a proosteogenic factor with well-established function on endothelial cells and osteoblasts 
during bone defect healing [2, 11,35]. Osteoblast lineage cell-derived VEGF has been reported as a key player in the stages of the bone repair process, i.e., osteogenic differentiation, angiogenesis and osteogenesis-angiogenesis coupling [11]. Hypoxia mimicking biomaterials reportedly promote bone defect healing via the upregulation of VEGF signaling $[8,36]$. Wang et al. reported that STAT3 signaling mediates VEGF production in MSCs [37]. In this study, we found that cellular hypoxia enhanced VEGF expression and the STAT3 inhibitor reversed this effect (Figs. $1 \mathrm{~h}$ and $3 \mathrm{~h}$ ). These results indicate the possible role of STAT3 signaling in the VEGF-mediated angiogenesis and the bone defect healing process. However, further in vitro and in vivo study focusing on the interaction between STAT3 and VEGF signaling during hypoxia-induced bone defect healing is necessary to prove this hypothesis.

Disrupted blood circulation creates a hypoxic environment in the bone defect area. Hypoxia increases HIF-1 $\alpha$ protein expression in precursor cells in the bone defect [38]. We found that HIF- $1 \alpha$ expression was upregulated in the injured femur, and STAT3 inhibitor reversed this effect (Figs. 5 and 7). Interestingly, expression of the early osteogenesis marker ALP was upregulated in the injured femur. The $\mathrm{CoCl}_{2}$ treatment further upregulated ALP expression and inhibition of STAT3 nullified this effect (Figs. 5 and 7). Moreover, STAT3 phosphorylation was upregulated in precursor cells in the defect area at weeks 3 and 5, and STAT3 inhibitor reversed this effect (Fig. 7). $\mathrm{CoCl}_{2}$ treatment enhanced new bone formation and bone defect healing, and inhibition of STAT3 reduced this effect.

Most of our results from in vitro studies were supported by the results from the in vivo study on bone defect healing. Activation of HIF-1 $\alpha$ in osteoblast lineage cells had been reported to enhance bone regeneration [6]. Similarly, Durand et al. reported that hypobaric hypoxia accelerates bone defect healing in mice [7]. STAT3 activation in mesenchymal stem cells had been reported to enhance osteogenic differentiation and in vivo bone formation [39-41]. Moreover, STAT3 activation in peripheral blood mononuclear cells had been reported to promote bone fracture healing [19]. Our findings show that hypoxia promotes osteogenesis and bone defect healing via activation of STAT3 signaling in precursor cells.

In this study, we investigated the effect of different durations of cellular hypoxia on osteogenic marker expression at the mRNA and protein levels. More prominent osteogenic marker ALP staining, ALP activity, and matrix mineralization were also investigated. The possible role of STAT3 signaling in hypoxia-mediated osteogenesis was investigated both in vitro and in vivo. In terms of future verification, there are a few possible approaches. We used $\mathrm{CoCl}_{2}$ to simulate hypoxia in vitro, and these results could be verified in the future with cell cultures incubated in a hypoxic environment. The results from mice MSCs should be verified with human MSCs or MSCs from STAT3 knockout mice. Similarly, a future study using MSC-specific STAT3 knockout mice for bone defect healing is recommended.

\section{Conclusions}

Both hypoxia and STAT3 signaling are involved in the osteogenic differentiation of precursor cells and bone defect healing. However, the role of the interaction between hypoxia and STAT3 signaling in bone defect healing is not clear. In this study, we found that cellular hypoxia inhibited MSC proliferation but enhanced osteogenic 
differentiation. Hypoxia for 3 days showed the highest anabolic effect on the osteogenic differentiation of MSCs. Hypoxia upregulates STAT3 phosphorylation and VEGF expression in MSCs. The STAT3 inhibitor reversed this effect. Hypoxia facilitated bone regeneration and bone defect healing in mouse femur bone defects. Inhibition of STAT3 signaling reduced the hypoxia-induced osteogenic differentiation of MSCs in vitro, and bone regeneration and healing in mice femoral defects, suggesting a possible role of STAT3 signaling in hypoxia-mediated osteogenic differentiation of precursor cells and bone defect healing.

\section{Supplementary information}

Supplementary information accompanies this paper at https://doi.org/10.1186/s11658-019-0191-8.

Additional file 1: Figure S1. Quantitative analysis of western blots for protein expression in BMSCS on day 7 (Fig. 2e). A - Col1a1. B - RUNX2. C - ALP. D - OSX. H1, H3, H5 and H7 represent hypoxia for 1, 3, 5 and 7 days, respectively. Inhibitor: STAT3 inhibitor. Data of quantitative analysis are the means \pm SD from 5 independent experiments, $n=5$. Significant effect of treatment, ${ }^{*} p<0.05,{ }^{* *} p<0.01{ }^{* * *} p<0.001$ compared with the control group. Figure S2. Quantitative analysis of western blots for protein expression in BMSCs after $3 \mathrm{~h}$ of culture (Fig. 3a). A - HIF-1a/GAPDH. B - pSTAT3/tSTAT3. Data of quantitative analysis are the means \pm SD from 3 independent experiments, $n=5$. Inhibitor: STAT3 inhibitor. Significant effect of treatment, ${ }^{*} p<0.05,{ }^{* *} p<0.01{ }^{* * *} p<$ 0.001 compared with the control group; ${ }^{\# \#} p<0.001$ compared with the $\mathrm{CoCl}_{2}$ group. Figure S3. Quantitative analysis of western blots for protein expression in BMSCs on day 7 (Fig. 4e). A - Col1a1. B - RunX2. C - ALP. D - OsX. $\mathrm{H1}, \mathrm{H} 3, \mathrm{H} 5$ and $\mathrm{H} 7$ represent hypoxia for 1, 3, 5 and 7 days, respectively. Inhibitor: STAT3 inhibitor. Data of quantitative analysis are the means \pm SD from 5 independent experiments, $n=5$. Significant effect of treatment compared to the control group: ${ }^{*} p<0.05,{ }^{* *} p<0.01$ and ${ }^{* * *} p<0.001$; the $\mathrm{CoCl}_{2}$ group: ${ }^{* \# \#} p<0.001$; and the $\mathrm{CoCl}_{2}+$ inhibitor group: ${ }^{\& \&} p<0.001$. Figure S4. Representative X-Ray images of mice femurs with bone defects. Inhibitor: STAT3 inhibitor.

\section{Abbreviations}

BMSCs: Bone marrow stromal cells; CoCl 2 : Cobalt chloride; HIF-1a: Hypoxia-inducible-factor 1-alpha; JAK2: Janus kinase 2; MSCs: Mesenchymal stem cells; STAT3: Signal transducer and activator of transcription-3; VEGF: Vascular endothelial growth factor

\section{Acknowledgements}

Not applicable.

\section{Authors' contributions}

$Z \mathrm{~L}$ and JLP: Study design, data interpretation and manuscript revision; $X Y$ and QW: in vitro and in vivo experiments and preparation of the first draft of the manuscript; XLY and YC: data collection and statistical analysis. All authors gave approval for the submission of this manuscript.

\section{Funding}

This study was supported by the funds of the National Natural Science Foundation of China (grant nos. 81470718, 81100736 and 81771051). The funding bodies have no role in study design, data collection, analysis and interpretation, and manuscript writing.

\section{Availability of data and materials}

All the data are included within this manuscript. The raw data of the study are available from the corresponding author on reasonable request.

\section{Ethics approval and consent to participate}

All the animals in this study were treated according to internationally recognized guidelines on animal welfare and with the approval of the Medical Ethics Committee of the Hospital of Stomatology of Wuhan University (approval number S07915050a, date: 2015-05-15).

\section{Consent for publication}

Not applicable.

\section{Competing interests}

The authors declare that they have no competing interests.

\section{Author details}

${ }^{1}$ The State Key Laboratory Breeding Base of Basic Science of Stomatology (Hubei-MOST) and Key Laboratory of Oral Biomedicine, Ministry of Education, School and Hospital of Stomatology, Wuhan University, 237 Luoyu Road, Wuhan 430079, China. 'Department of Oral and Maxillofacial Trauma and Plastic Surgery, School and Hospital of Stomatology, Wuhan University, 237 Luoyu Road, Wuhan 430079, China. ${ }^{3}$ Department of Stomatology, Union Hospital, Tongji Medical College, Huazhong University of Science and Technology, Wuhan 430022, China. ${ }^{4}$ Key Laboratory of Oral 
Medicine, Guangzhou Institute of Oral Disease, Affiliated Stomatology Hospital of Guangzhou Medical University, Guangzhou 510140, China.

Received: 26 July 2019 Accepted: 21 November 2019

Published online: 03 December 2019

\section{References}

1. Riddle RC, Khatri R, Schipani E, Clemens TL. Role of hypoxia-inducible factor-1alpha in angiogenic-osteogenic coupling. J Mol Med. 2009;87(6):583-90.

2. Schipani E, Maes C, Carmeliet G, Semenza GL. Regulation of osteogenesis-angiogenesis coupling by HIFs and VEGF. J Bone Miner Res. 2009;24(8):1347-53.

3. Wang Y, Wan C, Deng L, Liu X, Cao X, Gilbert SR, et al. The hypoxia-inducible factor alpha pathway couples angiogenesis to osteogenesis during skeletal development. J Clin Invest. 2007;117(6):1616-26.

4. Hung SP, Ho JH, Shih YR, Lo T, Lee OK. Hypoxia promotes proliferation and osteogenic differentiation potentials of human mesenchymal stem cells. J Orthop Res. 2012;30(2):260-6.

5. Zhang QB, Zhang ZQ, Fang SL, Liu YR, Jiang G, Li KF. Effects of hypoxia on proliferation and osteogenic differentiation of periodontal ligament stem cells: an in vitro and in vivo study. Genet Mol Res. 2014;13(4):10204-14.

6. Wan C, Gilbert SR, Wang Y, Cao X, Shen X, Ramaswamy G, et al. Activation of the hypoxia-inducible factor-1alpha pathway accelerates bone regeneration. Proc Natl Acad Sci U S A. 2008;105(2):686-91.

7. Durand M, Collombet JM, Frasca S, Begot L, Lataillade JJ, Le Bousse-Kerdiles MC, et al. In vivo hypobaric hypoxia performed during the remodeling process accelerates bone healing in mice. Stem Cells Transl Med. 2014;3(8):958-68.

8. Perez RA, Kim JH, Buitrago JO, Wall IB, Kim HW. Novel therapeutic core-shell hydrogel scaffolds with sequential delivery of cobalt and bone morphogenetic protein-2 for synergistic bone regeneration. Acta Biomater. 2015;23:295-308.

9. Drager J, Harvey EJ, Barralet J. Hypoxia signalling manipulation for bone regeneration. Expert Rev Mol Med. 2015;17:e6.

10. Fan LH, Li J, Yu ZF, Dang XQ, Wang KZ. The hypoxia-inducible factor pathway, Prolyl hydroxylase domain protein inhibitors, and their roles in bone repair and regeneration. Biomed Res Int. 2014;239356. https://doi.org/10.1155/2014/ 239356.

11. Hu K, Olsen BR. Osteoblast-derived VEGF regulates osteoblast differentiation and bone formation during bone repair. J Clin Invest. 2016;126(2):509-26.

12. Yan $Y$, Chen $H$, Zhang H, Guo C, Yang K, Chen $K$, et al. Vascularized 3D printed scaffolds for promoting bone regeneration. Biomaterials. 2019;190-191:97-110.

13. Jia $\mathrm{P}$, Chen $\mathrm{H}$, Kang H, Qi J, Zhao P, Jiang M, et al. Deferoxamine released from poly (lactic-co-glycolic acid) promotes healing of osteoporotic bone defect via enhanced angiogenesis and osteogenesis. J Biomed Mater Res A. 2016;104(10): 2515-27.

14. Darnell JE Jr. STATs and gene regulation. Science. 1997;277(5332):1630-5.

15. Stepkowski SM, Chen W, RosS JA, Nagy ZS, Kirken RA. STAT3: an important regulator of multiple cytokine functions. Transplantation. 2008;85(10):1372-7.

16. Liu F, Woitge HW, Braut A, Kronenberg MS, Lichtler AC, Mina M, et al. Expression and activity of osteoblast-targeted Cre recombinase transgenes in murine skeletal tissues. Int J Dev Biol. 2004;48(7):645-53.

17. Welte T, Zhang SS, Wang T, Zhang Z, Hesslein DG, Yin Z, et al. STAT3 deletion during hematopoiesis causes Crohn's disease-like pathogenesis and lethality: a critical role of STAT3 in innate immunity. Proc Natl Acad Sci U S A. 2003;100(4):1879-84.

18. Yu X, Wan Q, Cheng G, Cheng X, Zhang J, Pathak JL, et al. CoCl2 , a mimic of hypoxia, enhances bone marrow mesenchymal stem cells migration and osteogenic differentiation via STAT3 signaling pathway. Cell Biol Int. 2018; 42(10):1321-9.

19. Sun G, Wang Z, Ti Y, Wang Y, Wang J, Zhao J, et al. STAT3 promotes bone fracture healing by enhancing the FOXP3 expression and the suppressive function of regulatory T cells. APMIS. 2017;125(8):752-60.

20. Zhou H, Newnum AB, Martin JR, Li P, Nelson MT, Moh A, et al. Osteoblast/osteocyte-specific inactivation of Stat3 decreases load-driven bone formation and accumulates reactive oxygen species. Bone. 2011;49(3):404-11.

21. Yu X, Li Z, Wan Q, Cheng X, Zhang J, Pathak JL, et al. Inhibition of JAK2/STAT3 signaling suppresses bone marrow stromal cells proliferation and osteogenic differentiation, and impairs bone defect healing. Biol Chem. 2018;399(11):1313-23.

22. Wan QL, Schoenmaker T, Jansen IDC, Bian ZA, de Vries TJ, Everts V. Osteoblasts of calvaria induce higher numbers of osteoclasts than osteoblasts from long bone. Bone. 2016;86:10-21.

23. Yuan Y, Hilliard G, Ferguson T, Millhorn DE. Cobalt inhibits the interaction between hypoxia-inducible factor-alpha and von Hippel-Lindau protein by direct binding to hypoxia-inducible factor-alpha. J Biol Chem. 2003;278(18):15911-6.

24. Liu K, Li DQ, Huang XY, Lv K, Ongodia D, Zhu LL, et al. A murine femoral segmental defect model for bone tissue engineering using a novel rigid internal fixation system. J Surg Res. 2013;183(2):493-502.

25. Osathanon T, Vivatbutsiri P, Sukarawan W, Sriarj W, Pavasant P, Sooampon S. Cobalt chloride supplementation induces stem-cell marker expression and inhibits osteoblastic differentiation in human periodontal ligament cells. Arch Oral Biol. 2015;60(1):29-36.

26. Xu N, Liu H, Qu F, Fan J, Mao K, Yin Y, et al. Hypoxia inhibits the differentiation of mesenchymal stem cells into osteoblasts by activation of notch signaling. Exp Mol Pathol. 2013;94(1):33-9.

27. Yang DC, Yang MH, Tsai CC, Huang TF, Chen YH, Hung SC. Hypoxia inhibits osteogenesis in human mesenchymal stem cells through direct regulation of RUNX2 by TWIST. PLoS One. 2011;6(9):e23965.

28. Genetos DC, Toupadakis CA, Raheja LF, Wong A, Papanicolaou SE, Fyhrie DP, et al. Hypoxia decreases sclerostin expression and increases Wnt signaling in osteoblasts. J Cell Biochem. 2010;110(2):457-67.

29. Araldi E, Schipani E. Hypoxia, HIFs and bone development. Bone. 2010;47(2):190-6.

30. Daly AC, Sathy BN, Kelly DJ. Engineering large cartilage tissues using dynamic bioreactor culture at defined oxygen conditions. J Tissue Eng. 2018;9:2041731417753718.

31. Zhu XH, Yan HC, Zhang J, Qu HD, Qiu XS, Chen LA, et al. Intermittent hypoxia promotes hippocampal neurogenesis and produces antidepressant-like effects in adult rats. J Neurosci. 2010;30(38):12653-63. 
32. Feng $X$, Shen $S$, Cao $P$, Zhu $L$, Zhang $Y$, Zheng $K$, et al. The role of oncostatin $M$ regulates osteoblastic differentiation of dental pulp stem cells through STAT3 pathway. Cytotechnology. 2016;68(6):2699-709.

33. Gao W, McCormick J, Connolly M, Balogh E, Veale DJ, Fearon U. Hypoxia and STAT3 signalling interactions regulate proinflammatory pathways in rheumatoid arthritis. Ann Rheum Dis. 2015;74(6):1275-83.

34. Huang C, Ness VP, Yang X, Chen H, Luo J, Brown EB, et al. Spatiotemporal analyses of Osteogenesis and angiogenesis via Intravital imaging in cranial bone defect repair. J Bone Miner Res. 2015;30(7):1217-30.

35. Stegen S, van Gastel N, Carmeliet G. Bringing new life to damaged bone: the importance of angiogenesis in bone repair and regeneration. Bone. 2015;70:19-27.

36. Yao Q, Liu Y, Tao J, Baumgarten KM, Sun H. Hypoxia-mimicking Nanofibrous scaffolds promote endogenous bone regeneration. ACS Appl Mater Interfaces. 2016;8(47):32450-9.

37. Wang M, Zhang W, Crisostomo P, Markel T, Meldrum KK, Fu XY, et al. STAT3 mediates bone marrow mesenchymal stem cell VEGF production. J Mol Cell Cardiol. 2007;42(6):1009-15.

38. Kolar P, Gaber T, Perka C, Duda GN, Buttgereit F. Human early fracture hematoma is characterized by inflammation and hypoxia. Clin Orthop Relat Res. 2011;469(11):3118-26.

39. Nicolaidou V, Wong MM, Redpath AN, Ersek A, Baban DF, Williams LM, et al. Monocytes induce STAT3 activation in human mesenchymal stem cells to promote osteoblast formation. PLoS One. 2012;7(7):e39871.

40. Xie Z, Tang S, Ye G, Wang P, Li J, Liu W, et al. Interleukin-6/interleukin-6 receptor complex promotes osteogenic differentiation of bone marrow-derived mesenchymal stem cells. Stem Cell Res Ther. 2018;9(1):13.

41. Roberts SJ, Owen HC, Tam WL, Solie L, Van Cromphaut SJ, Van den Berghe G, et al. Humanized culture of periosteal progenitors in allogeneic serum enhances osteogenic differentiation and in vivo bone formation. Stem Cells Transl Med. 2014;3(2):218-28

\section{Publisher's Note}

Springer Nature remains neutral with regard to jurisdictional claims in published maps and institutional affiliations.

Ready to submit your research? Choose BMC and benefit from:

- fast, convenient online submission

- thorough peer review by experienced researchers in your field

- rapid publication on acceptance

- support for research data, including large and complex data types

- gold Open Access which fosters wider collaboration and increased citations

- maximum visibility for your research: over $100 \mathrm{M}$ website views per year

At $\mathrm{BMC}$, research is always in progress.

Learn more biomedcentral.com/submissions 